\title{
Monomer-Addition Growth with a Slow Initiation Step: A Growth Model for Silica Particles from Alkoxides
}

\author{
THEMIS MATSOUKAS AND ERDOGAN GULARI ${ }^{1}$ \\ Department of Chemical Engineering, The University of Michigan, Ann Arbor, Michigan 48109
}

Received January 27, 1988; accepted November 4, 1988

\begin{abstract}
A simplified monomer-addition model with a first-order activation step is developed to describe the dynamics of growth of silica particles from alkoxides. In the limit of slow hydrolysis, we obtain expressions for the evolution of the particle mass and particle polydispersity, as well as an expression for the particle size as a function of the hydrolysis rate constant, the polymerization rate constant, and the initial concentration of the orthosilicate. We find that the formation of the particles is adequately modeled by a reaction limited growth. (c) 1989 Academic Press, Inc.
\end{abstract}

\section{INTRODUCTION}

Hydrolysis and polymerization of silicon alkoxides in the presence of ammonia lead to the formation of a stable suspension of particles as opposed to gels obtained in the presence of an acid (1). The chemical reactions can be schematically represented as

$$
\begin{aligned}
& \mathrm{Si}(\mathrm{OR})_{4}+x \mathrm{H}_{2} \mathrm{O} \rightarrow \\
& \mathrm{Si}(\mathrm{OH})_{4-x}+x \mathrm{ROH} \quad \text { (Hydrolysis) } \\
& \equiv \mathrm{Si}-\mathrm{OH}+\mathrm{OH}-\mathrm{Si} \equiv \rightarrow \\
& \equiv \mathrm{Si}-\mathrm{O}-\mathrm{SI} \equiv+\mathrm{H}_{2} \mathrm{O}
\end{aligned}
$$

(Polymerization),

where OR is an alkoxy group, most often the methoxy or ethoxy derivative. Interest in the ammonia-catalyzed system lies in the fact that the produced particles are spherical in shape and exhibit a remarkably narrow size distribution $(2,3)$. Furthermore, by controlling the concentration of ammonia it is possible to form particles with sizes that cover almost the entire colloidal range. These characteristics are desirable in powder and ceramics technology as well as in the production of well-defined model systems for colloidal studies and instrument calibration.

\footnotetext{
${ }^{1}$ To whom correspondence should be addressed.
}

Stoeber et al. (2) and Van Helden et al. (3) have reported detailed studies on particle morphology and size distribution as a function of the reactants. The size is mostly sensitive to the concentration of ammonia. As the concentration of ammonia increases larger particles are formed and so it is possible to produce particles from $20 \mathrm{~nm}$ to about $2 \mu \mathrm{m}$ in diameter.

In terms of growth dynamics, hydrolysis plays an important role. It is a slow process and as shown in our previous paper (4) it is the rate-limiting step. Under conditions of excess water concentration it is a first-order reaction in the alkoxide and is accelerated as the ammonia concentration is increased. The goal in this work is to formulate a quantitative approach to the growth and identify the factors that affect the size and polydispersity of the particles.

A picture of this process can be constructed on the basis of some general arguments. The stability of the particle suspension is accounted for by electrostatic effects. The surface absorbs $\mathrm{OH}^{-}$ions and the charge prevents interparticle bonding (1). This can be demonstrated by addition of salt which induces aggregation, occasionally leading to a gel. The absence of aggregation partly explains the narrow size distribution. The rate of nucleation is another 
factor with a strong effect on the particle distribution. In our previous work (4) we found that nucleation takes place for a limited period of time in the early stage of the process. Eventually, growth becomes the dominant process and the number of particles remains constant as the rate of nucleation decays to insignificant levels. This result reflects the dynamic competition between nucleation and growth. A key factor that controls the balance between the two competing processes is hydrolysis, the chemical step that releases the active monomer. In light of these observations the growth can be described as a monomer-addition process in the presence of a rate-controlling activation reaction (hydrolysis).

Monomer-addition models, a special case of the more general aggregation processes, have generated considerable interest, primarily because of the very good characteristics of the morphology and polydispersity of the resulting particles. Of particular interest is the question whether growth is reaction- or diffusion-limited. Because the two mechanisms predict a different dependence of the growth rate upon the particle size, they also have a different effect on both the final size of the particles and their polydispersity. In a related chemical system, Santacesaria et al. (5) used a reaction-limited model to describe the thermal precipitation of $\mathrm{Ti}_{2} \mathrm{O}$ from titanium sulfate. This process leads to the formation of small particles that eventually agglomerate to form a wide distribution of aggregates. Jean and Ring (6), on the other hand, found that the formation of large titania particles $(0.5-1.0 \mu \mathrm{m}$ in diameter) from the alkoxide was diffusion-limited, and this conclusion was supported by the time evolution of the polydispersity.

Keefer (7) used a Monte Carlo model to study the structure of the particles. He found that fully hydrolyzed species give rise to uniformly compact (nonfractal) particles with smooth boundaries. Partly hydrolyzed species, however, produced progressively irregular boundaries. His SAXS results indicated a transition from mass fractals to surface fractals with a decreasing concentration of water.
These findings provide useful information on the processes that control the morphology of the particle, but questions such as those concerning the effect of the dynamic competition between nucleation and growth, a process that controls the final size and the polydispersity of the particles, have not been addressed.

Hydrolysis has a direct effect on the final size. In the absence of such a step, the numerical solution of the growth equations for diffusion-limited growth showed (8) that at steady state, the particle distribution consists of oligomers with an average molecular weight of about 3. This is a result of the fact that any monomer in this process can act as a nucleation site. Large particles cannot be formed because monomers are consumed primarily in the nucleation process. The picture is quite different in the presence of a slow monomeractivation step. Only the hydrolyzed monomers are available for nucleation and the slow release of the monomer acts as a mechanism that inhibits nucleation and, therefore, it promotes growth of large particles.

Solutions to problems more relevant to the silica system were developed by Hendriks (9), who considered the monomer-addition problem with a variety of initial conditions for the monomer, including fixed initial concentration, a source of constant strength, and an infinite pool of monomer. His results show the effect of different growth mechanisms on the average size and the higher moments of the distribution. The silica system, described by a time-dependent source of the monomer with a slow exponential decay (first-order hydrolysis), does not fall in the above categories.

In the following sections we formulate a monomer-addition model in the presence of a first-order initiation step and investigate the effect upon the steady-state mean size and polydispersity of the particles. The behavior of the system depends strongly upon the reaction kernel $\kappa_{i}$, i.e., the rate constant for the reaction between a monomer and an $i$-mer. We consider two cases: (i) reaction-limited growth which is characterized by a strong size dependence of the kernel on the size $(10,11)$, 
and (ii) diffusion-limited growth with much weaker size dependence $(8,11)$. We emphasize that the terms reaction- and diffusion-limited growth refer to the actual growth mechanism, i.e., the bonding between a monomer and a particle, although the overall rate may be enveloped by the rate-limiting hydrolysis. For the above cases we develop relations between the particle size and the hydrolysis rate constant $\kappa_{0}$. We also derive asymptotic expressions for the evolution of the polydispersity, and we compare the predictions to available experimental data.

\section{THE MODEL}

Let $c_{i}$ denote the concentration of species with molecular weight $i$ (in units of the monomer), $c_{i}$ the concentration of the unhydrolyzed monomer. Hydrolysis produces the active monomer which subsequently reacts with either active monomers (nucleation) or with polymeric species $C_{i}$ to produce $C_{i+1}$ (growth). The model is based on the irreversible steps

$$
\begin{aligned}
C_{0} \stackrel{k_{\mathrm{h}}}{\rightarrow} C_{1} & \text { (Hydrolysis) } \\
2 C_{1} \stackrel{k_{1}}{\rightarrow} C_{2} & \text { (Nucleation) } \\
C_{1}+C_{i} \stackrel{k_{i}}{\rightarrow} C_{i+1} & \text { (Growth). }
\end{aligned}
$$

Assuming elementary steps, the equations that describe these reactions are in dimensionless form

$$
\begin{gathered}
\frac{d n_{0}}{d t}=-\kappa_{0} n_{0} \\
\frac{d n_{1}}{d t}=\kappa_{0} n_{0}-2 \kappa_{1} n_{1}^{2}-n_{1} \sum_{i \geqslant 2} \kappa_{i} n_{i} \\
\frac{d n_{i}}{d t}=\kappa_{i-1} n_{i-1} n_{1}-\kappa_{i} n_{i} n_{1},
\end{gathered}
$$

where $n_{i}=c_{i} / c, \kappa_{i}=k_{i} / k_{\mathrm{p}}, \kappa_{0}=k_{\mathrm{h}} / k_{\mathrm{p}} c_{0}, t$ $=c_{0} k_{\mathrm{p}} \tau, \tau$ is real time, and $c_{0}$ is the initial concentration of the unhydrolyzed monomer. We have assumed here that $k_{i}$, the rate constant for the production of $i$-mers, is written in the form

$$
k_{i}=k_{\mathrm{p}} \kappa_{i},
$$

where $k_{\mathrm{p}}$ is the intrinsic rate constant and $\kappa_{0}$ is a dimensionless function of the particle size $i$. Usually $k_{\mathrm{p}}$ is normalized so that $\kappa_{1}=1$ but we have chosen instead to let $\kappa_{1}$ arbitrary in order to investigate the effect of the nucleation rate constant to the particle size. It can be easily verified that Eqs. [1a]-[1c] satisfy the mass conservation which is expressed as

$$
\frac{d}{d t}\left(n_{0}+\sum_{i=1}^{\infty} i n_{i}\right)=0 .
$$

We define the $m$ th-order moment of the particle distribution as

$$
M_{m}=\sum_{i \geqslant 2} i^{m} n_{i}
$$

Note that the definition refers to the particles, that is, species with a molecular weight of 2 or higher. Neither the unhydrolyzed nor the active monomer is included. We choose this definition because we want to focus on the particle distribution. In addition, this definition is consistent with what we observe in light scattering experiments. The intensity scattered by the particles scales as the square of the particle mass, thus weighing the larger particles more heavily than the smaller ones. As a result, the monomer is virtually invisible in both static and dynamic light scattering when sizable particles (in the $\mathrm{nm}$ range) have been formed. Following the definition, $M_{0}$ is the number of the growing particles, $M_{1}$ their mass, and $M_{2}$ the second moment of the distribution which scales as the intensity of the scattered light. Multiplying Eq. [1c] by $i^{m}$ and summing over all $i \geqslant 2$ we obtain the equation for $M_{m}$;

$\frac{d M_{m}}{d t}=2^{m} \kappa_{1} n_{1}^{2}$

$$
+\sum_{i \geqslant 2}\left[(i+1)^{m}-i^{m}\right] \kappa_{i} n_{i} n_{1}
$$

\subsection{Growth Kernels}

In order to proceed with the solution of these equations it is necessary to establish a

Journal of Colloid and Interface Science, Vol. 132, No, 1, October 1, 1989 
functional dependence between the growth reaction rate and the size of the particle. It is typical for growth processes to assume a power-law dependence of the form $\kappa_{i}=i^{a}(9$, 10). On grounds of physical arguments (10) we expect the exponent $\alpha$ to lie in the interval $0 \leqslant a \leqslant 1$. The constant kernel $(a=0)$ corresponds to a growth process whose rate is independent of the particle size. Such is the case of linear-polymer growth ( fixed number of reaction sites per particle). The proportional kernel $(a=1)$ describes a process where all the monomers within a particle (or a fixed fraction thereof) are potential sites for growth. Real cases lie in between. Two cases are of particular interest, reaction- and diffusionlimited growth. In a reaction-limited process the growth rate is limited by the bonding reaction and is proportional to the number of possible bonding configurations between the monomer and the particle. The growth kernel scales as the particle surface area and for smooth particles $a=\frac{2}{3}$. However, any surface roughness with fractal properties will push this number closer to 1 (7). In the diffusion-limited process the growth rate is limited by the collision frequency between a monomer and a particle. In this case the growth kernel is given by $(8,11)$

$$
\kappa_{i}=\left(r_{1}+r_{i}\right)\left(D_{1}+D_{i}\right),
$$

where $r_{1}, r_{i}$ are the radii of a monomer and an $i$-mer, $D_{1}$ and $D_{i}$ are their respective diffusivities. Using the Stokes-Einstein relation $r_{i} D_{i}=$ const (8), we can distinguish two cases and further simplify the kernel. At short times $i$ is not significantly larger than 1 and we can approximate $\kappa_{i} \approx 1$ or $a=0$. At longer times, $i \gg 1$ (also $r_{i} \gg r_{1}$ and $D_{i} \ll D_{1}$ ), therefore

$$
\kappa_{i} \approx D_{1} r_{i} \sim r_{i} \sim i^{1 / 3} \text {. }
$$

Fractional powers, however, introduce noninteger moments and analytical handling of the equations is not as simple.

In the following sections we investigate the behavior of the reaction system in the hydrolysis-limited regime $\left(\kappa_{0} \ll 1\right)$ for the cases of $a=1$ and $a=0$ as approximations to reaction- and diffusion-limited growth, respectively. Aside from the mathematical simplification, there is additional justification. While the proportional kernel overestimates the strength of the reaction-limited process, it retains the characteristic strong size dependence and it is a reasonable approximation for the early stage of the growth when the oligomeric species that act as nuclei have sufficiently open structure. The constant kernel underestimates the strength of the diffusion-limited process, but it is not in large error during the early stages of the growth, or for distributions with only moderate polydispersity.

The simplification to the system of the differential equations is considerable. First we note that for integer values of $m$, Eqs. [1b] and [4] take the form

$$
\begin{aligned}
\frac{d n_{1}}{d t} & =k_{0} n_{0}-2 \kappa_{1} n_{1}^{2}-n_{1} M_{a} \\
\frac{d M_{m}}{d t} & =2^{m} \kappa_{1} n_{1}^{2}+n_{1} \sum_{l=0}^{m-1}\left(\begin{array}{l}
m \\
l
\end{array}\right) M_{l+a} .
\end{aligned}
$$

More specifically, for $a=1$ and $a=0$ the infinite set of Eqs. [1] is reduced to a set involving $n_{0}, n_{1}$, and a finite number of the lower integer moments.

\subsection{The Proportional Kernel $\left(a=1, \kappa_{i}=i\right)$}

In this case the equations of interest take the form

$$
\begin{aligned}
& \frac{d n_{0}}{d t}=-\kappa_{0} n_{0} \\
& \frac{d n_{1}}{d t}=\kappa_{0} n_{0}-2 \kappa_{1} n_{1}^{2}-n_{1} M_{1} \\
& \frac{d M_{0}}{d t}=\kappa_{1} n_{1}^{2} \\
& \frac{d M_{1}}{d t}=2 \kappa_{1} n_{1}^{2}+n_{1} M_{1} \\
& \frac{d M_{2}}{d t}=4 \kappa_{1} n_{1}^{2}+n_{1} M_{1}+2 n_{1} M_{2}
\end{aligned}
$$

with initial conditions $n_{0}(0)=1, n_{1}(0)$ $=M_{0}(0)=M_{1}(0)=M_{2}(0)=0$. 
2.2.a. Size of the particles. We are interested in obtaining a relationship between the final particle size $r$ and the hydrolysis constant $\kappa_{0}$ when hydrolysis is the rate-limited step ( $\kappa_{0}$ $\ll 1)$. The equation for $n_{0}$ is not coupled to the rest and it yields the solution $n_{0}$ $=\kappa_{0} e^{-\kappa_{0} t}$. The mass balance takes the form $n_{0}$ $+n_{1}+M_{1}$ and can also be written as

$$
\begin{gathered}
n_{1}+M_{1}=\phi \\
\phi=1-e^{-\kappa_{0} t} .
\end{gathered}
$$

When $\kappa_{0} \ll 1, \phi$ changes slowly in time and we can approximately write

$$
\begin{aligned}
\phi d t & \approx d \phi t \\
\frac{d n_{1}}{\phi} & \approx d\left(\frac{n_{1}}{\phi}\right) .
\end{aligned}
$$

Initially the concentration of the active monomer $n_{1}$ increases but it must eventually fall as growth becomes the dominant process. This transient becomes faster as the process is further pushed in the hydrolysis-limited regime. $M_{0}$ depends on $n_{1}$ only; therefore we expect the evolution of both $M_{0}$ and $n_{1}$ to be fast and essentially completed during the early period of the process, during which the following approximations are valid:

$$
\begin{aligned}
\phi & \approx \kappa_{0} t \\
\frac{\kappa_{0} e^{-\kappa_{0} t}}{\phi^{2}} & \approx \frac{1}{\phi t} .
\end{aligned}
$$

We divide both parts of Eq. [10b] by $\phi^{2}$ and making use of the above approximations we finally have

$$
\begin{aligned}
\frac{d\left(n_{1} / \phi\right)}{d(\phi t)}=-2 \kappa_{1} & \left(\frac{n_{1}}{\phi}\right)^{2} \\
& -\frac{n_{1}}{\phi}\left(1-\frac{n_{1}}{\phi}\right)+\frac{1}{\phi t},
\end{aligned}
$$

the solution of which must be of the form $n_{1}$ / $\phi=F(\phi t)$ or $n_{1}=\phi F(\phi t)$, where $F$ is a function which depends on $\kappa_{1}$, but not explicitly on $\kappa_{0}$. With this expression for $n_{1}$ we can integrate Eq. [10c] for $M_{0}$ to obtain

$$
M_{0}=\int_{0}^{t} \kappa_{1} n_{1}^{2} d t=\int_{0}^{t} \kappa_{1} \phi^{2} F^{2}(\phi t) d t .
$$

We let $z=\phi t(z \rightarrow \infty$ as $t \rightarrow \infty)$. For short times $\phi \approx \sqrt{\kappa_{0} z}$ and finally we get for $M_{0}$

$$
M_{0}=\sqrt{\kappa_{0}} \int_{0}^{z} \kappa_{1} \sqrt{z} F^{2}(z) d z .
$$

At steady state $M_{0} \sim \sqrt{\kappa_{0}}$ since the integral on the right-hand side calculated from 0 to infinity is a constant independent of $\kappa_{0}$. Because $F(z)$ depends upon $\kappa_{1}$ we cannot obtain a relationship between the number of the particles and the nucleation rate constant.

We also note that $1 / M_{0}$ is the number average size of the particles at steady state $\left(M_{1}(\infty)=1\right)$. At steady state $M_{0} r^{3} \sim M_{1}=1$ and the final particle size $r$ is related to the dimensionless hydrolysis constant $\kappa_{0}$ through

$$
r \sim\left(\frac{1}{\kappa_{0}}\right)^{1 / 6}=\left(\frac{k_{\mathrm{p}} c_{0}}{k_{\mathrm{h}}}\right)^{1 / 6} .
$$

2.2.b. First moment. Making use of Eq. [11a] the equation for $M_{1}$ becomes

$\frac{d M_{1}}{d t}=\left(\phi-M_{1}\right)\left(2 \kappa_{1}+\left(1-2 \kappa_{1}\right) M_{1}\right)$.

We use the same approximations as above (Eqs. [12a] and [12b]) and after integration we finally obtain for $M_{1}$

$$
\begin{aligned}
M_{1} & =\phi W(\phi t) \\
W(\phi t) & =\frac{1-e^{-\phi t}}{1+\left(\left(1-2 \kappa_{1}\right) / 2 \kappa_{1}\right) e^{-\phi t}} .
\end{aligned}
$$

When $\kappa_{0} \ll 1, e^{-\phi t} \rightarrow 0$. Therefore, $W(\phi t) \rightarrow 1$ and $M_{1} \approx \phi=1-e^{-\kappa_{0} t}$; i.e., the rate of growth is asymptotically equal to the rate of monomer release, as we would expect for the hydrolysis-limited case.

\subsection{The Constant Kernel $\left(a=0, k_{i}=1\right)$}

In this case the equations read

$$
\begin{aligned}
& \frac{d n_{0}}{d t}=-\kappa_{0} n_{0} \\
& \frac{d n_{1}}{d t}=\kappa_{0} n_{0}-2 \kappa_{1} n_{1}^{2}-n_{1} M_{0}
\end{aligned}
$$




$$
\begin{aligned}
& \frac{d M_{0}}{d t}=\kappa_{1} n_{1}^{2} \\
& \frac{d M_{1}}{d t}=2 \kappa_{1} n_{1}^{2}+n_{1} M_{0} \\
& \frac{d M_{2}}{d t}=4 \kappa_{1} n_{1}^{2}+2 n_{1} M_{1}+n_{1} M_{0}
\end{aligned}
$$

with initial conditions $n_{0}(0)=1, n_{1}(0)$ $=M_{0}(0)=M_{1}(0)=M_{2}(0)=0$.

2.3.a. Size of the particles. Here we follow a different procedure. When $\kappa_{0} \ll 1$ the concentration of the active monomer is essentially zero (the monomer reacts as soon as it is produced). We can write then

$$
\frac{d n_{1}}{d t} \approx 0
$$

to obtain an algebraic equation for $n_{1}$,

$$
2 \kappa_{1} n_{1}^{2}+n_{1} M_{0}-Q=0,
$$

where $Q=\kappa_{0} e^{-\kappa_{0} t}$. The concentration of the hydrolyzed monomer $n_{1}$ is given by the positive root

$$
n_{1}=\frac{-M_{0}+\sqrt{M_{0}^{2}+8 \kappa_{1} Q}}{4 \kappa_{1}} \approx \frac{Q}{M_{0}},
$$

where we used $\sqrt{M_{0}^{2}+\delta}-\sqrt{M_{0}^{2}} \approx \delta / 2 M_{0}, \delta$ $=8 \kappa_{1} Q \ll 1$, valid for $\kappa_{0} \ll 1$. We return the expression for $n_{1}$ to Eq. [20c] and after integration we obtain

$$
M_{0}(t)=\left(\frac{3}{2} \kappa_{0} \kappa_{1}\right)^{1 / 3}\left(1-e^{-\kappa_{0} t}\right)^{1 / 3} .
$$

We see that in the limit of very slow hydrolysis the number of particles $M_{0}$ has again a powerlaw dependence upon $\kappa_{0}$ but his time the exponent is equal to $\frac{1}{3}$. The corresponding relation for the particle radius is

$$
r \sim\left(\frac{1}{\kappa_{0}}\right)^{1 / 9}=\left(\frac{k_{\mathrm{p}} c_{0}}{k_{\mathrm{h}}}\right)^{1 / 9} .
$$

From Eq. [23] we also find that $M_{0} \sim \kappa_{1}^{1 / 3}$. As we would expect, larger particles are formed as the nucleation rate constant $\kappa_{1}$ becomes smaller.

We have verified these asymptotic relations numerically and we show the results in Fig. 1.

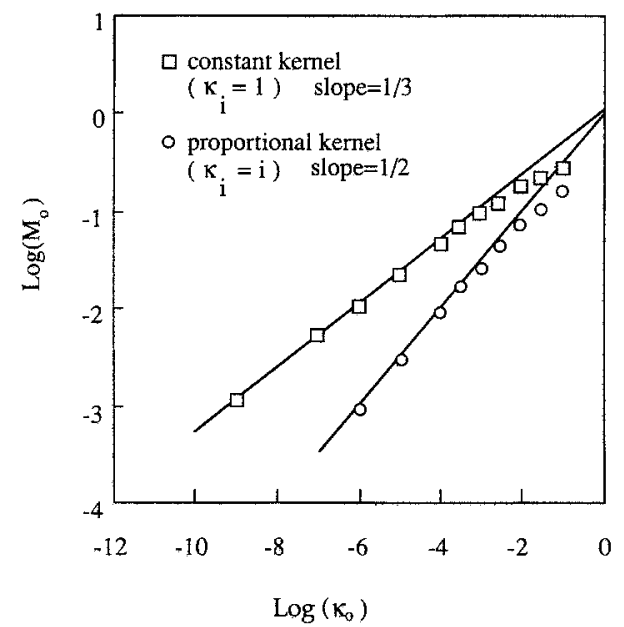

FIG. 1. The number of the particles $M_{0}$ as a function of the hydrolysis constant $\kappa_{0}=\kappa_{h} / k_{\mathrm{p}} c_{0}$ for $\kappa_{i}=i$ and $\kappa_{i}$ $=1$. Results obtined from numerical solution of Eqs. [8] and [24] with $\kappa_{1}=1$. In the limit of slow hydrolysis $\left(\kappa_{0} \rightarrow 0\right), M_{0}$ scales as $\kappa_{0}^{\lambda}$, with $\lambda=\frac{1}{3}$ for the constant kernel and $\lambda=\frac{1}{2}$ for the proportional kernel.

A point to observe is that for the same value of $\kappa_{0}$ the constant kernel produces smaller particles (larger $M_{0}$ ) than the proportional kernel.

2.3.b. First moment. The evolution of the particle mass may be obtained using Eqs. [21], [22] and the mass balance given by Eq. [11]. The result is

$$
M_{1}=\phi-\left(\frac{2}{3} \frac{\kappa_{0}^{2}}{\kappa_{1}}\right)^{1 / 3} \frac{e^{-\kappa_{0} t}}{\left(1-e^{-2 \kappa_{0} t}\right)^{1 / 3}} .
$$

As in the case of the proportional kernel, when $\kappa_{0} \ll 1, M_{1} \approx \phi=1-e^{-\kappa_{0} t}$; i.e., the growth rate is asymptotically equal to the rate of hydrolysis.

\subsection{Growth Kernels and Polydispersity}

When the particle size becomes appreciable, the constant and proportional kernels represent increasingly poorer approximations of diffusion- and reaction-limited growth, respectively. To assess the effect on the polydispersity, we must deal with the exact, noninteger kernels. In general, we assume that the 
long-time evolution of the variance $\sigma^{2}$ has a power-law dependence on the average size $\mu_{1}$,

$$
\sigma^{2} \sim \mu_{1}^{-\beta}
$$

where the normalized variance $\sigma^{2}$ is defined as

$$
\sigma^{2}=\frac{M_{0} M_{2}-M_{1}^{2}}{M_{1}^{2}}
$$

and the number average mass of the particle $\mu_{1}$ is given by $\mu_{1}=M_{1} / M_{0}$. For the special cases of $a=0$ and $a=1$, we can solve Eqs. [10d], [10e] and [20d], [20e] to find $\beta=1$ and $\beta=0$, respectively. In the case of diffusionlimited growth we find (12)

$$
a=\frac{1}{3}, \quad \beta \approx 1, \quad \sigma^{2} \sim \mu_{1}^{-1} \sim r^{-3},
$$

a result virtually identical to that of the constant kernel. Similarly, for the reaction-limited model we find

$$
a=\frac{2}{3}, \quad \beta \approx \frac{2}{3}, \quad \sigma^{2} \sim \mu_{1}^{-2 / 3} \sim r^{-2} .
$$

From Eqs. [27] and [28] we see that in both reaction- and diffusion-limited growth the polydispersity decreases with increasing size but with a different exponent. We may utilize this result in order to identify the growth kernel during the later stages of the growth.

\section{DISCUSSION}

The major difficulty in experimentally assessing the validity of these models comes from the fact that growth is limited by the slow hydrolysis. In such a case the total particle mass $M_{1}$ evolves as a first-order process with a time constant equal to that of hydrolysis independently of the actual growth mechanism, as shown by Eqs. [19] and [24]. For moderate polydispersity, the second moment is approximately proportional to the square of the total particle mass $M_{1}$. The evolution of the lower moments is insensitive to the reaction kernel $\kappa_{i}$ and, consequently, dynamic experiments of the type we reported earlier (4) do not actually provide useful information to discriminate between the two mechanisms. Equations [17] and [23] on the other hand are open to experimental testing. While it is true that $k_{\mathrm{p}}$ and $k_{\mathrm{h}}$ cannot be varied independently or in a well- defined manner (ammonia, for instance, affects both rate constants), we can vary $c_{0}$, the initial concentration of the alkoxide, and observe the effect upon the final size of the particles. The results are shown in Fig. 2 for different concentrations of ammonia. Particles were grown in ethanol in a procedure similar to that described in our earlier paper (4) and sizes were calculated using dynamic light scattering. We see that the solid lines which are drawn with a slope of $\frac{1}{6}$ provide a good description of the dependence well for a 10 -fold change in $c_{0}$, in a range recommended by Stoeber et al. (2) for the production of a narrow distribution. Given the simplicity of the model and the fact that no fitting of adjustable parameters is involved, we conclude that the proportional kernel describes the formation of the particles fairly well.

At this point we cannot assess the sensitivity of our results to noninteger values of $a$. In the general case $a$ should be of the form $a=d_{\mathrm{s}}$ / $3(10)$, where $d_{s}$, the surface fractal dimension, is bounded by $2 \leqslant d_{s} \leqslant 3$. When the particle surface is smooth, $d_{\mathrm{s}}=2$ in which case $a=\frac{2}{3}$.

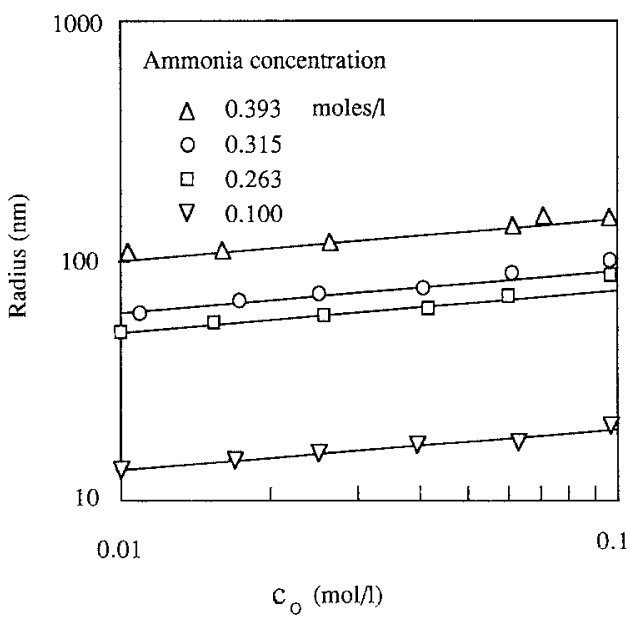

FIG. 2. Size of the particles as a function of the initial concentration of the alkoxide for different concentrations of ammonia. The solid lines are drawn with a slope of $\frac{1}{6}$ predicted by Eq. [15] for reaction-limited growth of nuclei with fairly open structure $(a=1)$. The concentration of $\mathrm{H}_{2} \mathrm{O}$ was $5.5 \mathrm{M}$, in large excess above the $4: 1$ stoichiometric ratio in order to assure pseudo-first-order kinetics of the hydrolysis.

Journal of Colloid and Interface Science, Vol. 132, No. 1, October 1, 1989 
Electron micrographs show the larger particles ( 100 to $300 \mathrm{~nm}$ in diameter) to be nearly perfectly spherical with smooth boundaries. The situation with smaller particles is, however, different. Keefer (7) measured the surface fractal dimension $d_{\mathrm{s}}$ of small silica particles (less than $25 \mathrm{~nm}$ in diameter) grown under water-deficient conditions and obtained values in the range 2.5 to 2.8 . These correspond to values of $a$ from 0.8 to 0.9 . The limiting value of $a=1$ corresponds to totally "transparent" particles, in which the monomer can penetrate freely and react at any length. This case must not be totally dismissed as physically unreal. Its applicability can be argued for the early part of the process, when small nuclei such as cyclic oligomers (1), for instance, have a structure sufficiently open to be described by the proportional kernel.

Once the total number of particles reaches a steady state, growth continues by addition of monomer with no further nucleation. At this stage of the process, the growth kernel controls the distribution of mass among the particles and, thus, the polydispersity. We expect kernels that favor the larger particles more than the small ones to produce a wider distribution and indeed we observe that the exponent $\beta$, a measure of the sharpening of the distribution, becomes smaller as $a$ increases. In order to further test the applicability of the reaction-limited model, we followed the evolution of the particle distribution in time using TEM. Typically, 200-500 particles were used to calculate the average size and standard deviation. In Fig. 3 we plot the normalized variance as percentage standard deviation squared vs the particle size. In logarithmic axes we obtain a linear relationship with a slope of -1.75 \pm 0.2 , very close to the value of -2 predicted by the reaction-limited model and certainly above the value of -3 which corresponds to the diffusion-limited kernel.

Hydrolysis has a direct effect upon the final particle size and our results quantify this dependence that we expect intuitively: As long as monomers react much more quickly than they are produced, their concentration re-

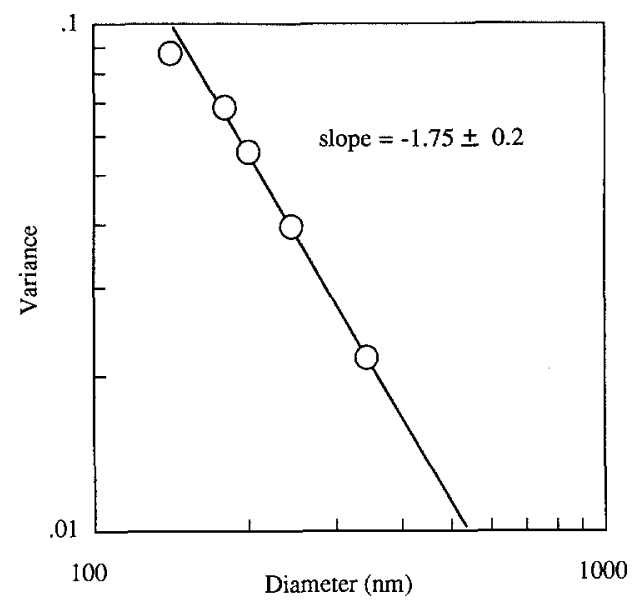

FIG. 3. Evolution of the variance of the particle population as a function of the average size during the growth. The variance has a power-law dependence on the size with an exponent $-1.75 \pm 0.2$, in reasonably good agreement with the predicted value of -2 for particles with smooth surface $\left(a=\frac{2}{3}\right)$. The particles were grown in ethanol from $0.044 M$ TEOS and $5.5 M \mathrm{H}_{2} \mathrm{O}$ in the presence of 0.315 $M \mathrm{NH}_{4} \mathrm{OH}$.

mains low. Therefore, growth, which is first order in $n_{1}$, is favored over nucleation, a second-order process. We can generalize these remarks and conclude that factors that inhibit hydrolysis, inhibit nucleation as well, and vice versa. In a sense, hydrolysis acts as a timeprogrammed monomer release, a process which is required in the production of powders with a narrow size distribution, because it provides an initial burst of nuclei that subsequently grow without further nucleation $(13,14)$.

Equation [17] also helps us to rationalize the effect of ammonia. It is known that hydrolysis kinetics are accelerated and larger particles are formed as the concentration of ammonia is increased. The obvious effect of ammonia is to accelerate hydrolysis, the ratelimiting step. On the other hand, it is known that the increased concentration of $\mathrm{OH}^{-}$catalyzes the growth reaction ( 1 ). In order to reconcile this fact with the observed size increase, we must conclude that while both $k_{\mathrm{p}}$ and $k_{\mathrm{h}}$ increase, their ratio actually decreases. In the framework of our dimensionless equations this 
means that the system is pushed further into the hydrolysis limited regime and the result, according to Eq. [17], is larger particles.

\section{CONCLUSIONS}

In the presence of hydrolysis as the ratelimiting step, the evolution of the mass of the particles, $M_{1}$ and their second moment, $M_{2}$, is not sensitive to the actual growth mechanism. The final particle size and the polydispersity, on the other hand, exhibit a distinct dependence upon the growth kernel and this can be used to experimentally identify the mechanism by which particle growth occurs.

The formulation of the proposed growth schemes is the simplest possible which contains the important characteristics of the process, while not introducing adjustable parameters. Simplified in many respects, this model based on monomer-addition growth in the presence of a slow initiation step (hydrolysis) provides a good basis for a semiquantitative description of the formation of silica particles from alkoxides in the presence of ammonia.

\section{APPENDIX: NOMENCLATURE}

a growth exponent of growth kernel

$c_{0} \quad$ initial concentration of unhydrolyzed monomer

$d_{\mathrm{s}} \quad$ surface fractal dimension

$k_{\mathrm{h}}, k_{\mathrm{p}}$ dimensional hydrolysis and polymerization rate constants

$M_{m} \quad m$ th-order moment of the particle distribution

$n_{0} \quad$ dimensionless concentration of unhydrolyzed monomer

$n_{i} \quad$ dimensionless concentration of particles of molecular weight $i$

$Q=\kappa_{0} e^{-\kappa_{0} t} \quad$ source term for the production of hydrolyzed monomer

$r \quad$ final particle radius

$t \quad$ dimensionless time

\section{Greek Symbols}

$\kappa_{0} \quad$ dimensionless hydrolysis constant

$\kappa_{i} \quad$ dimensionless rate constant of growth of an $i$-mer
$\mu_{1}=M_{1} / M_{0}$

$\phi=1-e^{-\kappa_{0} t}$

number average molecular weight of the particles

combined concentration of free $\left(n_{1}\right)$ and bound $\left(M_{1}\right)$ monomer

$\sigma^{2} \quad$ normalized variance of the particle size distribution

$\tau \quad$ real time

\section{ACKNOWLEDGMENTS}

We thank Dennis Vigil and Bob Ziff for their helpful comments; Ed McGrady and Kristen Fichthorn for critically reading this manuscript. Financial support for this work by the NSF under Grant CBT 8701781 is gratefully acknowledged. The analytical scanning transmission electron microscope used in this work was aquired under Grant DMR-77-09643 from the National Science Foundation.

\section{REFERENCES}

1. Iler, R., "The Chemistry of Silica." Wiley, New York, 1979.

2. Stoeber, W., Fink A., and Bohn, E., J. Colloid Interface Sci. 26, 62 (1968).

3. Van Helden, A. K., Jansen, J. W., and Vrij, A., $J$. Colloid Interface Sci. 81, 354 (1981).

4. Matsoukas, T., and Gulari, E., J. Colloid Interface Sci. 124, 252 ( 1988).

5. Santacesaria, E., Tonello, M., Storti, G., Pace R. C., and Carra, S., J. Colloid Interface Sci. 111, 44 (1985).

6. Jean, J. H., and Ring, T. A., Langmuir 2, 251 (1986).

7. Keefer, K. D., in "Better Ceramics through Chemistry, Mater. Res. Soc. Symp. Proc." (C. J. Brinker, D. E. Clark, and D. R. Ulrich, Eds.), Vol. 73, p. 79. Mater. Res. Soc., Pittsburgh, 1986.

8. Feder, $\mathbf{J}$., and Jøssang, $\mathbf{T}$., in "Scaling Phenomena in Disordered Systems" (R. Pynn and A. Skjeltorp, Eds.), p. 99. Plenum, New York, 1985.

9. Hendriks, E. M., J. Colloid Interface Sci. 97, 176 (1984).

10. Hendriks, E. M., Ernst, M. H., and Ziff, R. M., $J$. Stat. Phys. 31, 519 (1983).

11. Weitz, D. A., Huang, J. S., Ling, M. Y., and Sung, J., Phys. Rev. Lett. 54, 1416 (1985).

12. Matsoukas, T., and Gulari, E., manuscript in preparation.

13. Calvert, P., in "Better Ceramics through Chemistry, Mater. Res. Soc. Symp. Proc. (C. J. Brinker, D. E. Clark, and D. R. Ulrich, Eds.), Vol 73, p. 79. Mater. Res. Soc., Pittsburgh, 1986.

14. Overbeek, J. Th. G., Adv. Colloid Interface Sci. 15 , 251 (1982). 